\title{
Tablet Container
}

National Cancer Institute

\section{Source}

National Cancer Institute. Tablet Container. NCI Thesaurus. Code C149979.

Container without neck and with a flat bottom, suited for tablets, capsules, etc., can be re-closed well. 